\section{RESUSCITATION AND PERIVENTRICULAR/ INTRAVENTRICULAR HEMORRHAGE}

T. Nikolic ${ }^{1}$, B. Jankovic ${ }^{2}$, B. Otasevic ${ }^{3}$, V. Hajdarpasic ${ }^{3}$, V. Bankovic ${ }^{1}$

${ }^{1}$ Neonatology, Institute for Gynecology and Obstetrics, Clinical Center, ${ }^{2}$ Neonatology, Institute for Mother and Child, 3 Institute for Neonatology, Belgrade, Serbia

Aim: The aim of our study was to evaluate the role of resuscitation as the risk factor for periventricular/ intraventricular hemorrhage in preterm newborns.

Methods: The one year prospective study involved 164 premature newborns, gestational age 23 to 32 weeks, transported from maternity hospital to the Institute for neonatology. According to the development of $\mathrm{PVH} / \mathrm{IVH}$ three groups were formed. The first group (C- control) was formed of 39 newborns without $\mathrm{PVH} / \mathrm{IVH}$, second group (Mmild) involved 88 newborns with mild $\mathrm{PVH} / \mathrm{IVH}$ and the third group 37 newborns who developed severe $\mathrm{PVH} / \mathrm{IVH}$ (S- severe). The need for resuscitation was analyzed and compared between the groups, and the relative risk was calculated.

Results: The need for resuscitation using positive pressure ventilation (PPV) had $19(48.7 \%)$ newborn from group C, 45 newborns (58.1\%) from the group $M$ and the $29(79,4 \%)$ newborns from the group S. The difference was statistically significant, $\mathrm{P}<0.05$ (0.002). The difference was even more obvious $(P<$ 0.001 ) comparing the use of sodium bicarbonate in resuscitation. As the part of resuscitation $\mathrm{NaHCO}_{3}$ received $5(12.8 \%)$ newborns from the group C 8 $(9.1 \%)$ from the group $\mathrm{M}$ and $13(35.2 \%)$ from the group. Calculated relative risk (RR 95\% Cl)) for developing severe form of PVH/IVH was 2.12 (0.97 - 4.62) when PPV is performed and 2.87 (1.69 4.88) after the use of $\mathrm{NaHCO}_{3}$

Conclusion: Beside immaturity, the need for resuscitation may represent significant risk factor for the development of $\mathrm{PVH} / \mathrm{IVH}$ in premature newborns, especially if the $\mathrm{NaHCO}_{3}$ was used.

\section{BRAIN MONITORING OF NEWBORNS UNDERGOING CARDIAC SURGERY WITH CARDIOPULMONARY BYPASS (CBP)}

D. Blanco Bravo, C. Herbozo, I. Pescador, B. Bernardo, E. Maderuelo, P. Chimenti, M. Sanchez Luna

Hospital General Universitario Gregorio Marañon, Madrid, Spain

Aim: To study cerebral electrical activity and local regional oximetry during cardiopulmonary by-pass (CPB) and determine the periods of time in which the brain is at its most vulnerability.

Setting: Neonatal intensive care unit of a tertiary university-affiliated children's hospital.

Methods: Prospective observational study of newborns with congenital heart disease needing surgical therapy on CBP. Regional cerebral oxymetry (CrSO2) with near-infrared spectroscopy (NIRS, INVOS) and amplitude integrated electroencephalography (aEEG, CFM Olympic) were continuously monitored.

Results: 23 infants were studied. 19/23 underwent cardiac surgery on CPB with deep hypothermic circulatory arrest (DHCA), 2 had selective cerebral perfusion (SCP), 4/23 did not need circulatory arrest. $70.6 \%$ presented critical electric activity during circulatory arrest (aEEG) and a rapid fall in cerebral oxymetry (CrSO2 < 40\%) during CPB-DHCA. The electrical activity ceased when reentering cardiopulmonary bypass. In cases of CPB with SCP there was no critical electrical activity in the aEEG, nonetheless these patients had critical values of $\mathrm{CrSO} 2$ in the postoperative period.

Electrical seizures occurred in 19/23 patients (82.6\%). During anesthetic induction 2 patients presented seizures (8.7\%), 12 (52.2\%) during DHCA, $8(34.8 \%)$ during modified ultrafiltration (MUF), 7 (30.4\%) during re-warming and $4(17.4 \%)$ during the postoperative period. Only 4 patients $(17.4 \%)$ didn't have critical electrical activity at any time

Conclusions: Brain activity monitoring with aEEG allows us to identify seizures during CPB, which suggests an inadequate neuro-protection mostly during circulatory arrest.

The combined use of aEEG and INVOS allows a rapid detection of high-risk situations for neurological injury and low cardiac output. 